\title{
Dosimetric response of radiochromic films to protons of low energies in the Bragg peak region
}

\author{
M. C. Battaglia, ${ }^{1}$ D. Schardt, ${ }^{2}$ J. M. Espino, ${ }^{1,3}$ M. I. Gallardo, ${ }^{3}$ M. A. Cortés-Giraldo, ${ }^{3}$ \\ J. M. Quesada, ${ }^{3}$ A. M. Lallena, ${ }^{4}$ H. Miras, ${ }^{5}$ and D. Guirado ${ }^{6}$ \\ ${ }^{1}$ Centro Nacional de Aceleradores (CNA) de la Universidad de Sevilla, \\ Junta de Andalucía y CSIC, 41092 Seville, Spain \\ ${ }^{2}$ GSI Helmholtz Centre for Heavy Ions Research, 64291 Darmstadt, Germany \\ ${ }^{3}$ Departamento de Física Atómica, Molecular y Nuclear, Universidad de Sevilla, 41013 Seville, Spain \\ ${ }^{4}$ Departamento de Física Atómica, Molecular y Nuclear, Universidad de Granada, \\ E-18071 Granada, Spain \\ ${ }^{5}$ Hospital Universitario Virgen Macarena (HUVM), 41071 Seville, Spain \\ ${ }^{6}$ Servicio de Radiofísica y Protección Radiológica, Hospital Universitario San Cecilio, \\ E-18012 Granada, Spain
}

(Received 30 September 2015; published 21 June 2016)

\begin{abstract}
One of the major advantages of proton or ion beams, applied in cancer treatment, is their excellent depthdose profile exhibiting a low dose in the entrance channel and a distinct dose maximum (Bragg peak) near the end of range in tissue. In the region of the Bragg peak, where the protons or ions are almost stopped, experimental studies with low-energy particle beams and thin biological samples may contribute valuable information on the biological effectiveness in the stopping region. Such experiments, however, require beam optimization and special dosimetry techniques for determining the absolute dose and dose homogeneity for very thin biological samples. At the National Centre of Accelerators in Seville, one of the beam lines at the $3 \mathrm{MV}$ Tandem Accelerator was equipped with a scattering device, a special parallelplate ionization chamber with very thin electrode foils and target holders for cell cultures. In this work, we present the calibration in absolute dose of EBT3 films [Gafchromic radiotherapy films, http://www.ashland .com/products/gafchromic-radiotherapy-films] for proton energies in the region of the Bragg peak, where the linear energy transfer increases and becomes more significant for radiobiology studies, as well as the response of the EBT3 films for different proton energy values. To irradiate the films in the Bragg peak region, the energy of the beam was degraded passively, by interposing Mylar foils of variable thickness to place the Bragg peak inside the active layer of the film. The results obtained for the beam degraded in Mylar foils are compared with the dose calculated by means of the measurement of the beam fluence with an ionization chamber and the energy loss predicted by SRIM2008 code.
\end{abstract}

DOI: 10.1103/PhysRevAccelBeams.19.064701

\section{INTRODUCTION}

The use of proton beams to treat cancer has increased significantly in recent years [1]. This is due to a combination of two factors: on one hand, the evidence of the benefits of this type of therapy over conventional radiotherapy (which uses photons or electrons) and, on the other hand, the advent of new efficient and compact commercial equipments with affordable costs, which allowed many centers to be able to offer this treatment strategy. The relative biological effectiveness (RBE) of protons can reach high values only in very thin slices of the Bragg peak and the steep falling edge behind. However this high-RBE effect is diluted in the treatments of realistic volumes with

Published by the American Physical Society under the terms of the Creative Commons Attribution 3.0 License. Further distribution of this work must maintain attribution to the author $(s)$ and the published article's title, journal citation, and DOI. high-energy beams (due to the longitudinal straggling). Therefore, so far the proton therapy centers use an RBE value of 1.1 recommended by the International Commission on Radiation Units and Measurements (ICRU). To unveil this fact, it is important to perform dedicated measurements to investigate the RBE in thin slices at low proton energies. The $3 \mathrm{MV}$ Tandem Accelerator at National Centre of Accelerators (CNA) in Seville provides proton beams with a maximum of energy of $6 \mathrm{MeV}$, using the SNIC II source through cesium sputtering. Such energy is suitable to implement radiobiology studies. To perform such studies, a dedicated beam line is required [2], for which special beam optimization and dosimetric techniques are needed. The special configuration of the setup allows one to irradiate cells growing in monolayer cultures placed in Petri dishes and mounted at the exit of the beam line, orthogonally with respect to the beam axis. Forthcoming studies will investigate the DNA damage produced by low energy protons which will be 
compared to the photon damage at low doses. In order to provide accurate dose measurements at the sample position two methods were implemented and compared: (1) EBT3 film dosimeters which were calibrated with photons in the Sevilla University Hospital Virgen Macarena (HUVM) and (2) calculation of the dose by using proton fluence values based on charge measurements with a parallel-plate thin ionization chamber placed in front of the samples.

Up to now, few studies were conducted about the EBT3 film dosimetry with low energy protons [3], especially regarding its use for proton dosimetry in the Bragg peak region.

In this work, we present the modifications carried out on the beam line and, more extensively, the dosimetric studies performed in the preparation of future cell sample irradiations. Special emphasis will be given to the calibration, made in terms of absorbed dose, of the most recent model of radiochromic films (Gafchromic EBT3) for low energy protons and to the study of the dose delivery in the Bragg peak region.

\section{MATERIALS AND METHODS}

\section{A. Beam line}

In order to perform the dosimetric studies presented in this paper, it was necessary to install several new elements in one of the beam lines placed at the exit of the $3 \mathrm{MV}$ Tandem Accelerator. The beam line used (Fig. 1) is located at 30 degrees at the exit of the distributor magnet behind the tandem accelerator. It is equipped with a retractable Faraday Cup placed at the entrance of the beam line and two beam profile monitors (BPM1 and BPM2) which allow to check the beam profile in two positions along the beam line.

It is crucial then to obtain a homogeneous beam profile onto the sample surface. For this reason, behind the first BPM, a manipulator with Au targets was placed in order to scatter the beam. For preliminary tests, we used two Au targets of thickness 2.0 and $5.4 \mathrm{mg} / \mathrm{cm}^{2}$ (Fig. 2) and studied which one could fit better the performances required.

In Fig. 3, we show the raw response of the radiochromic film (placed in the sample holder), for the irradiations performed with the scattering foils. The data show the beam profile projected along the horizontal direction (x).

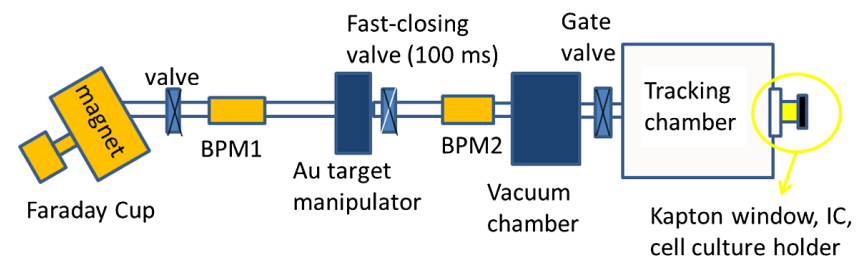

FIG. 1. Beam line scheme. The tracking chamber, installed in this line, was not used in these measurements.

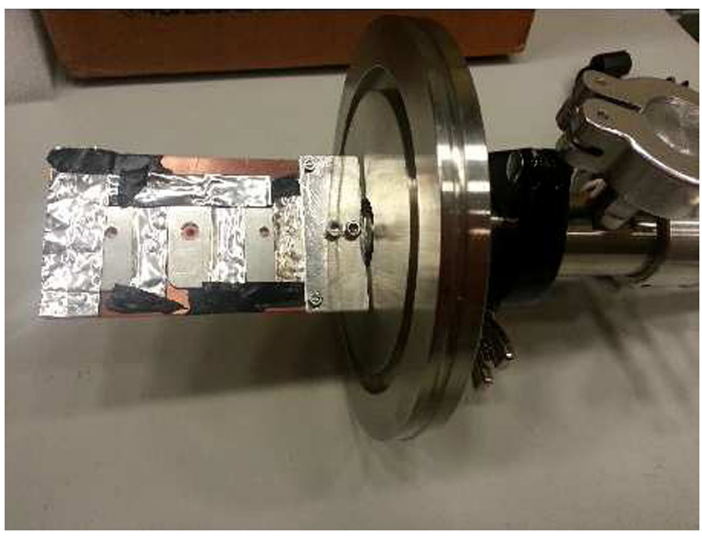

FIG. 2. Target manipulator with three positions. From right to left (always with a collimator $2 \mathrm{~mm}$ of diameter): no Au target; $2.0 \mathrm{mg} / \mathrm{cm}^{2} \mathrm{Au}$ target; $5.4 \mathrm{mg} / \mathrm{cm}^{2}$ Au target.

The maximum values correspond to the nonirradiated area, whereas the minimum corresponds to the irradiated ones. The structure of the holder of the films (with six Petri dishes) allows one to irradiate a circular area of $3.5 \mathrm{~cm}$ of
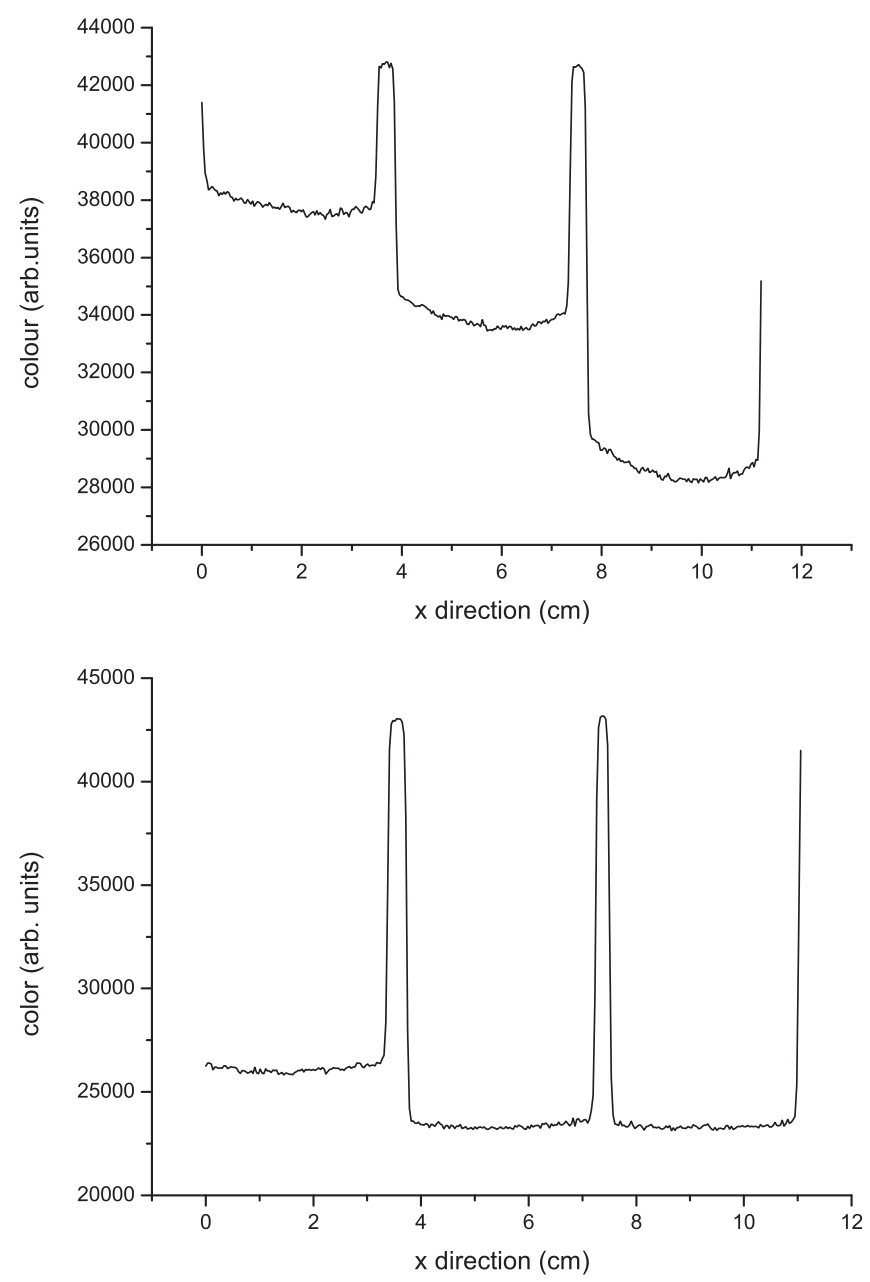

FIG. 3. Raw beam profiles obtained for 2.0 (top) and 5.4 $\mathrm{Au} \mathrm{mg} / \mathrm{cm}^{2}$ (bottom) targets. 


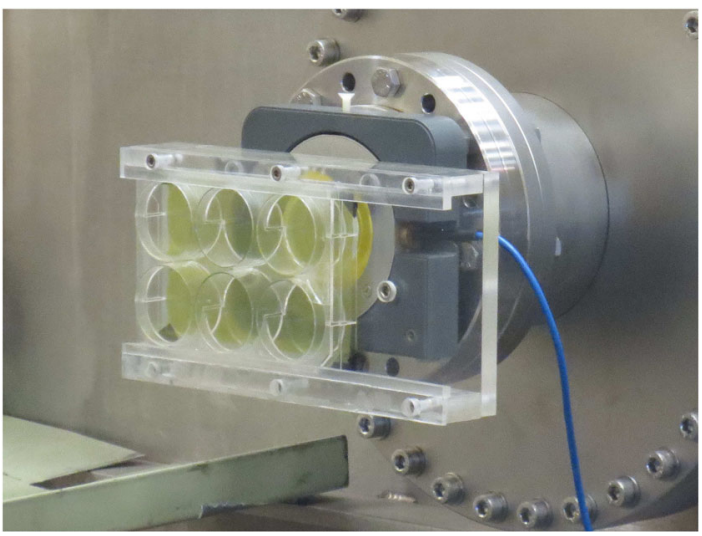

FIG. 4. Experimental setup for the irradiation of the radiochromic film. The picture shows the Kapton window, the IC and the holder for films mounted at the end of the beam line.

diameter. Figure 3 represents three irradiated areas projected along x. Good conditions in terms of beam energy loss and homogeneity were obtained using the gold target of $5.4 \mathrm{mg} / \mathrm{cm}^{2}$ thickness as scatterer.

In order to control the irradiation time a fast-closing pneumatic valve (100 ms closing time) was used as a beam shutter. A thin vacuum exit window made of Kapton (50 $\mu \mathrm{m}$ thick and $44 \mathrm{~mm}$ diameter) is placed at the end of the beam line, at a distance of $5155 \mathrm{~mm}$ from the gold foil, and mounted together with an ionization chamber (IC), fabricated at the GSI laboratory, consisting of three thin parallel electrodes with two air gaps in between, operated at $400 \mathrm{~V}$. A Keithley electrometer (model 6514) connected to the IC is used to measure the small charge (typically $4 \mathrm{nC}$ per cGy deposited) produced by the protons passing through the IC. A sample holder with six positions is placed directly behind the IC (Fig. 4). This holder is used to carry the Gafchromic EBT3 films and also for housing cell cultures. All materials traversed by the proton beam are listed in detail in Table I.

TABLE I. Elements and correspondent thickness of the beam line components. $\mathrm{N}$ indicates the order of the material layers as the protons pass through.

\begin{tabular}{lcc}
\hline \hline Target material & $\mathrm{N}$ & Thickness \\
\hline Gold scattering foil & 1 & $5.4 \mathrm{mg} / \mathrm{cm}^{2}$ \\
Kapton window & 2 & $50 \mu \mathrm{m}$ \\
Air gap & 3 & $10 \mathrm{~mm}$ \\
Three IC electrodes & $4,6,8$ & $7.5 \mu \mathrm{m}$ \\
Two IC air gaps & 5,7 & $6.75 \mathrm{~mm}$ \\
Air gap & 9 & $13 \mathrm{~mm}$ \\
EBT3 (polystyrene) & 10 & $125 \mu \mathrm{m}$ \\
EBT3 (lucyte) & 11 & $28 \mu \mathrm{m}$ \\
EBT3 (polystyrene) & 12 & $125 \mu \mathrm{m}$ \\
\hline \hline
\end{tabular}

\section{B. EBT3 Gafchromic film}

EBT3 Gafchromic films are the latest technology of radiochromic films, nowadays largely used for quality assurance in radiotherapy treatments [4]. Gafchromic films are made of clear, polyester outer layers which sandwich sensitive layers of microcrystalline diacetylene suspended in gelatin. Under exposure to ionizing radiation, they suffer a solid-state polymerization via a free-radical mechanism, exhibiting a blue color darkening. The optical density is correlated to the dose deposited in the sensitive layer (Fig. 5).

The Gafchromic EBT3 film is comprised of a single active layer $28 \mu \mathrm{m}$ thick of lucyte between two transparent polyester substrates of $125 \mu \mathrm{m}$ of thickness. Thanks to the stabilizers and other additives present in the active layer, the film shows a very small energy dependence $[5,6]$. These films have a special polyester substrate that prevents the formation of Newton's ring interference patterns in the images. The symmetric structure of the film eliminates the need for keeping track of which side of the film was placed on the scanner. The analysis is obtained using flatbed scanners. The film scanning must be performed always in the same direction since it influences the acquired image. As a self-developing film, EBT3 requires no postexposure processing and the film can be easily handled.

The software chosen to plot the beam profile was IMAGEJ, which is a public domain software written in Java for image processing. The image is uploaded in a 48-bit color mode, without any image correction, with 75 dpi of resolution and saved in the tiff uncompressed format.

Some of the most relevant characteristics of EBT3 films are as follows: (i) the response to film irradiation dose is somewhat different for each of the three color channels (red, green and blue; RGB) of the scanned image, making the analysis of one or another channel be the optimum for a given dose interval (the red channel is used for doses up to $10 \mathrm{~Gy}$, while the green one for doses above $40 \mathrm{~Gy}$; the blue channel shows a low sensitivity in the dose response and it is not used); (ii) small energy dependence for photons and electrons; (iii) close to tissue equivalence; (iv) high spatial resolution-can resolve features to at least $25 \mu \mathrm{m}$.

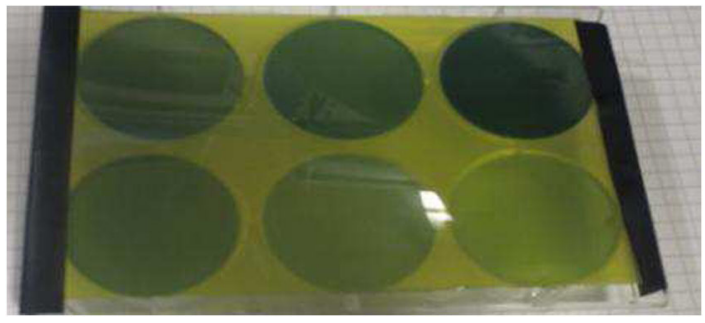

FIG. 5. EBT3 film irradiated with six different amounts of dose (0.5-4 Gy). 


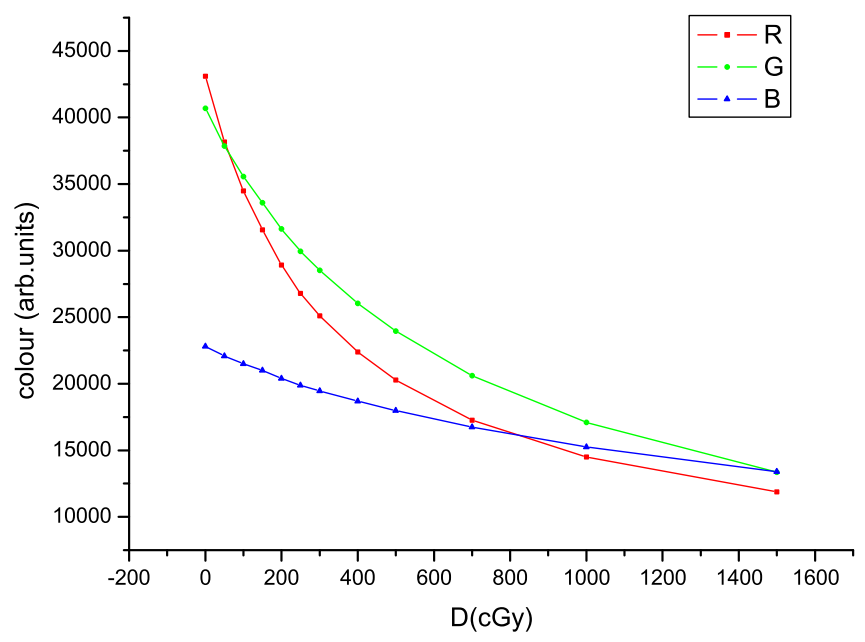

FIG. 6. Calibration of the EBT3 films in absolute dose for RGB channel colors.

\section{Calibration in absolute dose}

The calibration of the films was carried out at the University Hospital Virgen Macarena (HUVM) in Seville [7], using a $6 \mathrm{MV}$ photon beam produced by a linac accelerator. Under the condition of $100 \mathrm{~cm}$ of source to surface distance and $1.5 \mathrm{~cm}$ of solid water above the film, 100 monitor units delivered by the linac accelerator, correspond to $1 \mathrm{~Gy}$ of absorbed dose. The films were irradiated with different values of doses, and, subsequently, they were analyzed with the Epson perfection V700 photo scanner installed at CNA. The correlation between the intensity of the three color channels RGB of the scanned image and the dose represents the calibration curve (Fig. 6). As described previously, for the dose range used for radiobiology purposes, the red color channel is the most sensitive in terms of dose response [8]. Therefore, this channel was chosen to calibrate the films. In order to use the photon calibration also for protons, we assumed that for low-linear energy transfer (LET) protons (far from the Bragg peak) the film response to a given dose is the same as for photon irradiations with the same dose; thus, such calibration can be used for films irradiated with protons. This assumption is supported and verified by the measurements taken with the IC, as it is shown in the next section.

\section{IC measurements}

To irradiate the samples, it is important to achieve a homogeneous and uniform dose distribution in an area equal to the sample surface. So, the first step of the analysis is to check the uniformity for different values of dose. As shown in Fig. 7, by using the $\mathrm{Au}$ scattering foil of $5.4 \mathrm{mg} / \mathrm{cm}^{2}$, we are able to obtain a beam profile with a homogeneity better than $5 \%$.

The second step lies in the comparison between the dose values obtained with the calibrated films ( $\left.D_{\text {ЕBT3 }}\right)$ and those

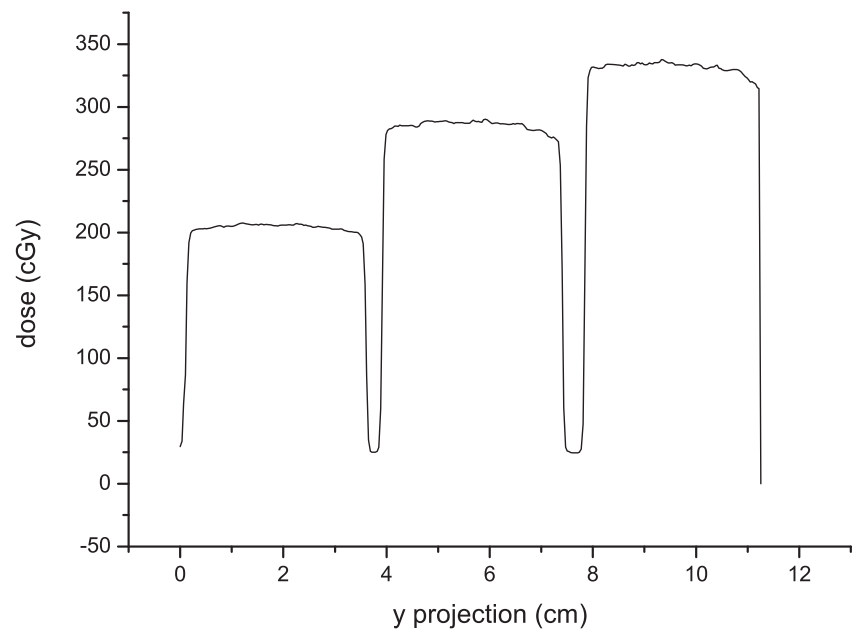

FIG. 7. Calibrated dose profile for three irradiations outside the Bragg peak region.

from the measurements of the beam fluence through the IC $\left(D_{\mathrm{FL}}\right)$. The dose $D_{\mathrm{FL}}$ in the film can be obtained as [9]

$$
D_{\mathrm{FL}}=F \frac{\mathrm{d} E}{\mathrm{~d} x} \frac{1}{\rho},
$$

where $F$ is the fluence of the protons through the IC, $\mathrm{d} E / \mathrm{d} x$ represents the stopping power of the protons integrated in the active layer of the film and $\rho$ is the density of the EBT3 active layer which is $1.2 \mathrm{~g} / \mathrm{cm}^{3}$ as given by the manufacturer; $\mathrm{d} E / \mathrm{d} x$ was computed by SRIM2008 code.

\section{RESULTS}

All the measurements were performed under the conditions of a nominal energy given by the accelerator fixed at $5.233 \mathrm{MeV}$ and low values of beam current of the order of $10 \mathrm{nA}$ before passing the Au scatterer.

The energy loss of the beam through the beam line elements was estimated using SRIM2008 code [10].

We can distinguish two main cases: the Bragg peak is placed at the second polyester substrate (Bragg peak outside the active layer, top of Fig. 8), or the maximum dose is placed in the active layer. The second case is critical, since the energy deposition presents a high gradient in a limited region, as shown in Fig. 8. In order to move the Bragg peak inside the active layer of the film, we studied the dose changes at different energies by interposing Mylar foils of different thicknesses. According to this, we define the Mylar thickness interval which placed the Bragg peak in the active layer as "Bragg peak region" $[26-51 \mu \mathrm{m}$ of Mylar, Figs. 8(b) and 8(c)]. By means of this technique, the energy of the beam was degraded passively maintaining the same accelerator and beam conditions for the whole series of measurements.

In Fig. 8(a) is shown the initial case when the Bragg peak lies clearly inside the second substrate of polystyrene. 

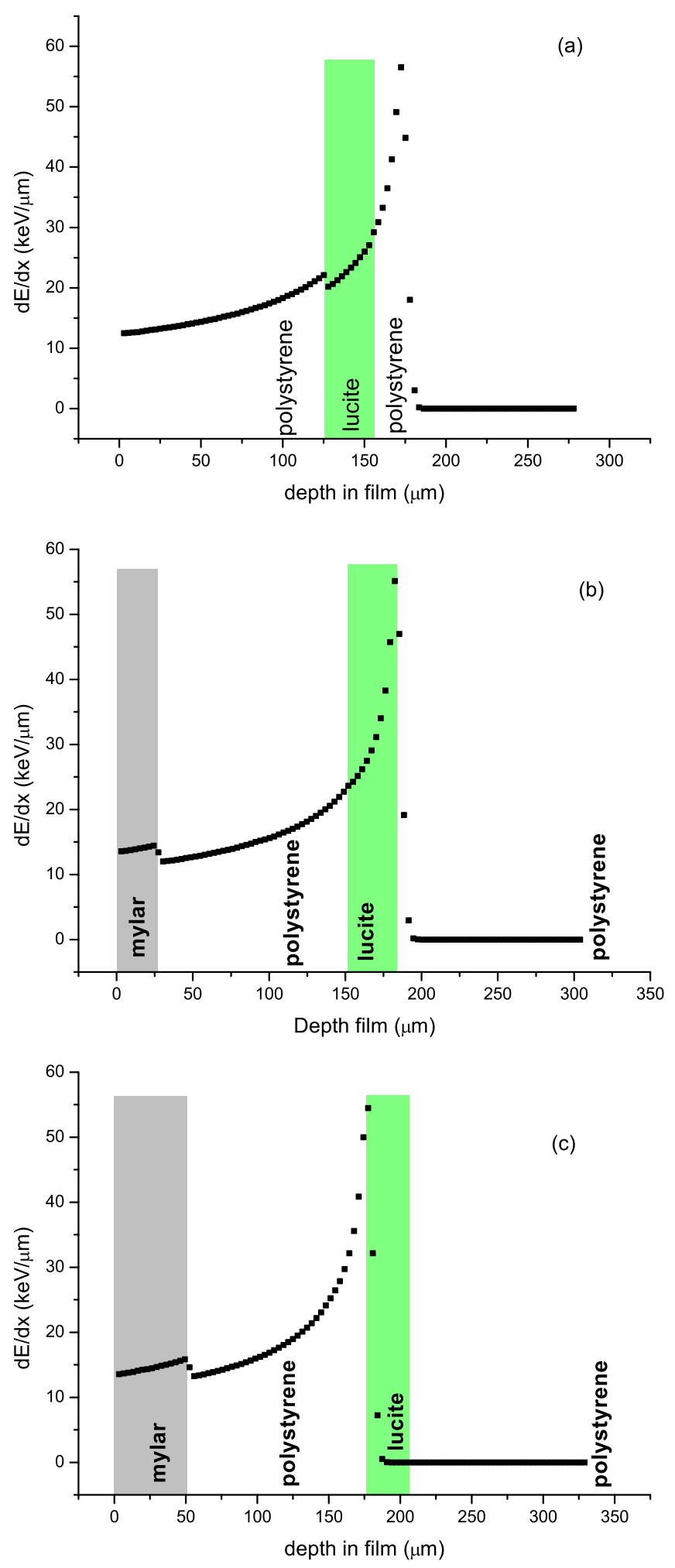

FIG. 8. Bragg curve inside the transversal section of the EBT3 film for beam energy degradation with (a) $0 \mu \mathrm{m}$, (b) $26 \mu \mathrm{m}$ and (c) $51 \mu \mathrm{m}$ of Mylar, placed between the IC and the first polyester substrate of the film.

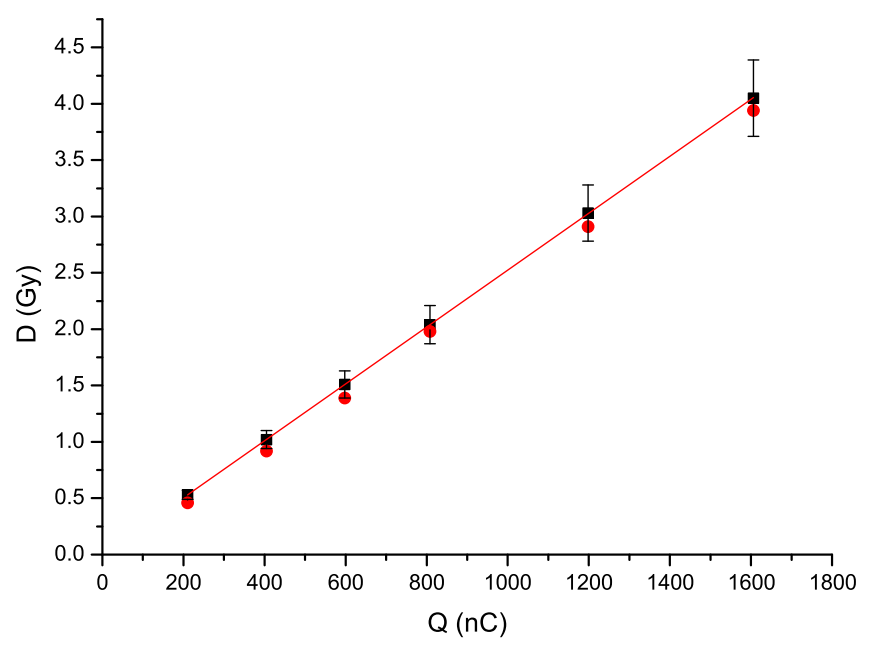

FIG. 9. Comparison of dose values obtained from EBT3 films (using the standard photon calibration, red circles) and calculated doses $\left(D_{\mathrm{FL}}\right)$, at the position of the active film zone, using proton fluences obtained from charge measurements with a parallel-plate IC in front of the film (black squares).

Then the LET in the active layer of protons is expected to be similar to that of photons and the absolute calibration is done as described above.

This assumption is also confirmed by the measurements taken with the IC (Fig. 9), which allow one to calculate the dose in the radiochromic film (based on the measured proton fluence). The results show for different values of dose that the ratio $D_{\mathrm{FL}} / D_{\mathrm{EBT} 3}$ keeps within the range $1.0-1.1$ in this region.

When the Bragg peak falls inside the active layer (central panel of Fig. 8), effects of saturation are evident. The darkening of the radiochromic films depends on the organic monomers, which polymerize under ionizing irradiation. When the Bragg peak is located inside the active layer, the LET and the associated stopping power increase abruptly, the energy of the protons is deposited in a small volume [11] and such an effect leads to a darkening of the film that is not anymore proportional to the absorbed dose. Moreover, previous studies showed that the film response is dependent on the proton energy near the Bragg peak and the distal falloff region [12-15].

Figure 10 presents the results obtained for eight irradiations where the initial energy of the protons delivered by the tandem accelerator was degraded using Mylar foils of 6 and $13 \mu \mathrm{m}$ (20\% of thickness tolerance, data reported in the manufacture guide) in different combinations (see Table II). In order to investigate the effect of saturation occurring in the EBT3 film when the Bragg peak approaches or falls inside the active area, Monte Carlo simulations (with SRIM2008 code) were performed to study how the dose changes by interposing Mylar foils of $2 \mu \mathrm{m}$ thick in a range 


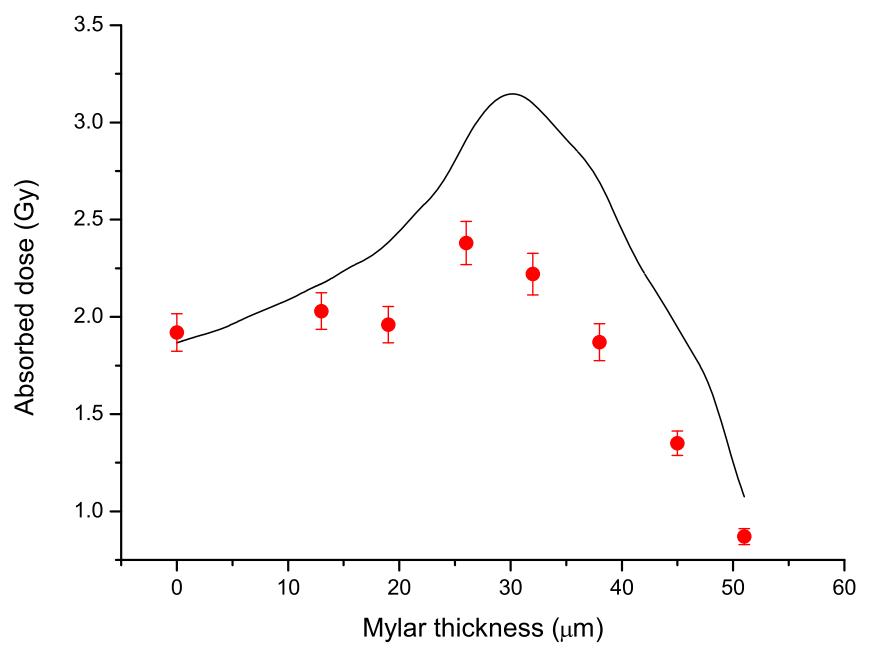

FIG. 10. Absorbed dose at different Mylar thickness. The black curve indicates SRIM simulations, while the red dots represent the experimental data of the calibrated dose for the EBT3. Around the maximum value that corresponds to a Mylar thickness of $32 \mu \mathrm{m}$, the film response is lower than expected because of the quenching effect.

of 2-50 $\mu \mathrm{m}$ (Fig. 10, black curve) and then compared to the experimental data of the film dose. This plot shows that the dose calculated by Eq. (1) (where the $\mathrm{d} E / \mathrm{d} x$ was computed with SRIM), deviates from the standard calibrated one, as soon as the Bragg peak approaches the active layer.

According to our measurements, the saturation effect starts for a Mylar foil with a thickness of $19 \mu \mathrm{m}$. In fact, the values reduce noticeably when the Mylar thickness ranges from few microns below the lowest limit of the Bragg peak region up to its upper limit, varying from $0.69 \pm 0.06$ to $0.82 \pm 0.07$. Looking at these results, we can make some important considerations: when the Bragg peak lies well outside of the active layer the film calibration can be used because the ratio $D_{\mathrm{EBT} 3} / D_{\mathrm{FL}}$ is compatible to 1 . By increasing the thickness of the Mylar foils, the Bragg peak is moved towards the active zone. A quenching effect can

TABLE II. Comparison between the dose calculated on the basis of proton fluences measured with the IC $\left(D_{\mathrm{FL}}\right)$ and the dose obtained from the film calibration with photons $\left(D_{\mathrm{EBT3}}\right)$, increasing the Mylar thickness.

\begin{tabular}{ll}
\hline \hline Mylar thickness $(\mu \mathrm{m})$ & $D_{\mathrm{EBT} 3} / D_{\mathrm{FL}}$ \\
\hline 0 & $1.03 \pm 0.09$ \\
13 & $0.93 \pm 0.08$ \\
19 & $0.82 \pm 0.07$ \\
26 & $0.81 \pm 0.07$ \\
32 & $0.71 \pm 0.06$ \\
38 & $0.69 \pm 0.06$ \\
45 & $0.71 \pm 0.06$ \\
51 & $0.81 \pm 0.07$ \\
\hline \hline
\end{tabular}

be noticed (between 19 and $51 \mu \mathrm{m}$ of Mylar in our measurements) with a marked effect when its maximum lies in the active zone (32-45 $\mu \mathrm{m}$ of Mylar). Finally, in the situation shown in Fig. 8(c), where the Bragg peak is just exiting the active area, the saturation effects are less evident due to the sharp decrease of the deposited dose. Other causes of uncertainties in the quantification of the quenching effect can be attributed to the stopping power libraries used by SRIM which show uncertainties of the order of $10 \%$ at energies in the proximity of the Bragg peak [16-18]. This source of uncertainty is also well known in other general purpose Monte Carlo codes such as PENH [19] and Geant4 [20].

\section{CONCLUSIONS}

In this work we present how a beam line has been prepared to get uniform spatial profile irradiation fields of low energy protons. The resulting setup is used to learn about the dose calibration of radiochromic films for proton energies at which maximum deposition occurs in their active layer. These studies can be important to supply an accessible way of measuring dose distributions in proton therapy cancer centers, since color changes in EBT3 can be associated to dose measurements. This is also of interest for the preparation of radiobiological experiments with proton beams.

It was possible to obtain a very homogeneous beam profile by scattering the protons onto a $\mathrm{Au}$ target of $5.4 \mathrm{mg} / \mathrm{cm}^{2}$. Our results show that the dose calibration protocol used at the HUVM for calibrating the EBT3 film under photon irradiation can be successfully adopted for low energy protons outside the Bragg peak region, as it has been verified through the IC fluence measurements. We were able to degrade passively the beam energy, maintaining the same beam optics for the whole set of measurements, while reaching small variations in the proton energy. The measurements in the Bragg peak region show the effects of saturation that occur when the LET increases. In this case, the ratio between the dose values obtained from the films and those calculated on the basis of the fluence measurements varies significantly. The quenching effect can be due to numerous factors and further studies will be necessary to establish the nature of such an effect and eventually to consider the possibility of using calibrated Gafchromic EBT3 films as dosimeters to measure the dose deposited inside the Bragg peak region.

\section{ACKNOWLEDGMENTS}

This work is supported by the EU Initial Training Marie Curie Network 289485 "Optimization of particle accelerators" (FP7-PEOPLE-2011-ITN-289485); by the Spanish Research Projects No. FPA2014-53290-C2-2-P and No. FPA2013-47327-C2-1-R; by the Andalusian Research Project No. P12-FQM-1605. 
[1] J. Maarten Schippers and M. Seidel, Operational and design aspects of accelerators for medical applications, Phys. Rev. ST Accel. Beams 18, 034801 (2015).

[2] M. Belli, R. Cherubini, G. Galeazzi, S. Mazzucato, G. Moschini, O. Sapora, G. Simone, and M. A. Tabocchini, Proton irradiation facility for radiobiological studies at a 7 MV Van De Graaff Accelerator, Nucl. Instrum. Methods Phys. Res., Sect. A 256, 576 (1987).

[3] M. Vadrucci et al., Calibration of GafChromic EBT3 for absorbed dose measurements in $5 \mathrm{MeV}$ proton beam and ${ }^{60}$ Co $\gamma$-rays, Med. Phys. 42, 4678 (2015).

[4] S. Devic, Radiochromic film dosimetry: Past, present, and future, Phys. Med. 27, 122 (2011).

[5] GAFCHROMIC EBT3 Scan Handling Guide, http://www .ashland.com/products/gafchromic-radiotherapy-films.

[6] I. Daftari, C. Castenadas, P. L. Petti, R. P. Singh, and L. J. Verhey, An application of GafChromic MD-55 film for 67.5 MeV clinical proton beam dosimetry, Phys. Med. Biol. 44, 2735 (1999).

[7] R. Arráns et al., Dosimetría con películas radiocrómicas, Rev. Fis. Med. 10, 83 (2009).

[8] J. Sorriaux et al., Evaluation of Gafchromic® EBT3 films characteristics in therapy photon, electron and proton beams, Phys. Med. 29, 599 (2013).

[9] G. Kraft, Tumor therapy with heavy charged particles, Prog. Part. Nucl. Phys. 45, S473 (2000).

[10] SRIM2008, http://www.oecd-nea.org/tools/abstract/detail/ nea-0919/.

[11] L. Zhao and I. J. Das, Gafchromic EBT film dosimetry in proton beams, Phys. Med. Biol. 55, N291 (2010).
[12] F. Fiorini, D. Kirby, J. Thompson, S. Green, D. J. Parker, B. Jones, and M. A. Hill, Dosimetry and spectral analysis of a radio-biological experiment using laser-driven proton beams, Phys. Med. 30, 454 (2014).

[13] S. Reinhardt, M. Würl, C. Greubel, N. Humble, J. J. Wilkens, M. Hillbrand, A. Mairani, W. Assmann, and K. Parodi, Investigation of EBT2 and EBT3 films for proton dosimetry in the $4-20 \mathrm{MeV}$ energy range, Radiat. Environ. Biophys. 54, 71 (2015).

[14] D. Kirby, S. Green, H. Palmans, R. Hugtenburg, C. Wojnecki, and D. Parker, LET dependence of GafChromic films and ion chamber in low-energy proton dosimetry, Phys. Med. Biol. 55, 417 (2010).

[15] A. Piermattei et al., Radiochromic film dosimetry of a low energy proton beam, Med. Phys. 27, 1655 (2000).

[16] W. Cayzac et al., Predictions for the energy loss of light ions in laser-generated plasmas at low and medium velocities, Phys. Rev. E 92, 053109 (2015).

[17] H. Paul and D. Sánchez-Parcerisa, A critical overview of recent stopping power programs for positive ions in solid elements, Nucl. Instrum. Methods Phys. Res., Sect. B 312, 110 (2013).

[18] ICRU Report 49, Stopping powers and ranges for protons and alpha particles, 1993.

[19] E. Sterpin, J. Sorriaux, and S. Vynckier, Extension of PENELOPE to protons: Simulation of nuclear reactions and benchmark with Geant4, Med. Phys. 40, 111705 (2013).

[20] S. Agostinelli et al., Geant4-a simulation toolkit, Nucl. Instrum. Methods Phys. Res., Sect. A 506, 250 (2003). 